\title{
Measurement of the cosmic-ray low-energy antiproton spectrum with the first BESS-Polar Antarctic flight
}

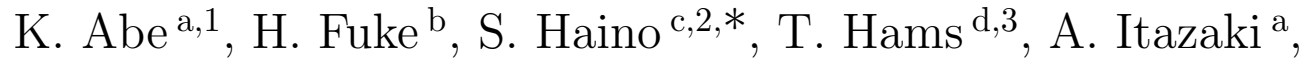 \\ K. C. Kim ${ }^{\mathrm{e}}$, T. Kumazawa ${ }^{\mathrm{c}}$, M. H. Lee ${ }^{\mathrm{e}}$, Y. Makida ${ }^{\mathrm{c}}$, \\ S. Matsuda ${ }^{c}$, K. Matsumoto ${ }^{c}$, J. W. Mitchell ${ }^{d}$, \\ A. A. Moiseev ${ }^{d}$, Z. Myers ${ }^{\mathrm{e}}{ }^{4}$, J. Nishimura $^{\mathrm{f}}$, M. Nozaki $^{\mathrm{c}}$, \\ R. Orito ${ }^{a, 5}$, J. F. Ormes ${ }^{\text {g }}$, M. Sasaki ${ }^{\mathrm{d}, 3}$, E. S. Seo ${ }^{\mathrm{e}}$, \\ Y. Shikaze ${ }^{\mathrm{a}, 6}$, R. E. Streitmatter ${ }^{\mathrm{d}}$, J. Suzuki ${ }^{\mathrm{c}}$, Y. Takasugi ${ }^{\text {a }}$, \\ K. Takeuchi ${ }^{\text {a }}$, K. Tanaka ${ }^{\mathrm{c}}$, T. Yamagami ${ }^{\mathrm{b}}$, A. Yamamoto $^{\mathrm{c}}$, \\ T. Yoshida ${ }^{b}, K$. Yoshimura ${ }^{c}$ \\ ${ }^{a}$ Kobe University, Kobe, Hyogo 657-8501, Japan \\ ${ }^{\mathrm{b}}$ Institute of Space and Astronautical Science, Japan Aerospace Exploration \\ Agency (ISAS/JAXA), Sagamihara, Kanagawa 229-8510, Japan \\ ${ }^{\mathrm{c}}$ High Energy Accelerator Research Organization (KEK), Tsukuba, Ibaraki \\ 305-0801, Japan \\ d National Aeronautics and Space Administration, Goddard Space Flight Center \\ (NASA/GSFC), Greenbelt, MD 20771, USA \\ e IPST, University of Maryland, College Park, MD 20742, USA \\ ${ }^{\mathrm{f}}$ The University of Tokyo, Bunkyo, Tokyo 113-0033 Japan \\ ${ }^{g}$ University of Denver, Denver, CO 80208, USA
}

\begin{abstract}
The BESS-Polar spectrometer had its first successful balloon flight over Antarctica in December 2004. During the 8.5-day long-duration flight, almost 0.9 billion events were recorded and 1,520 antiprotons were detected in the energy range $0.1-4.2 \mathrm{GeV}$. In this paper, we report the antiproton spectrum obtained, discuss the origin of cosmic-ray antiprotons, and use antiproton data to probe the effect of charge-signdependent drift in the solar modulation.
\end{abstract}

Key words: cosmic-ray antiproton, solar modulation, superconducting spectrometer

PACS: 95.85.Ry, 96.40.Kk, 98.70.Sa 


\section{Introduction}

Antiproton spectra have been measured by BESS (Balloon-borne Experiment with a Superconducting Spectrometer) in a series of flights from Lynn Lake, Canada providing reasonable statistics above $1 \mathrm{GeV}[1,2,3,4,5,6,7]$. Below 1 $\mathrm{GeV}$ statistics are limited and the effects of solar modulation are greater. The spectrum has a distinct peak around $2 \mathrm{GeV}$, showing the characteristic feature of secondary antiprotons produced by the interaction of Galactic cosmic-rays with the interstellar medium. The energy spectrum of these secondary antiprotons should decrease rapidly toward lower energies reflecting the kinematic constraints on antiproton production $[8,9,10]$ and toward higher energies reflecting the steep power-law spectra of primary particles producing the antiprotons. In addition to these secondary antiprotons, there might be a source of primary antiprotons. Such sources have been suggested to result from the evaporation of primordial black holes $(\mathrm{PBH})$ or from the annihilation of neutralino dark matter $[11,12,13,14]$. The spectrum of antiprotons from a primary source might have a peak in the energy region below $1 \mathrm{GeV}$, giving a flatter composite spectrum with excess flux compared to the purely secondary spectrum $[10,13]$. The influence of a low energy primary peak would be most evident at solar minimum $[12,13]$ and BESS $(1995+1997)$ measurements during this period [3,4] suggested this possibility. On the other hand, BESS-1998 [5] and subsequent measurements [6,7], which were taken after the solar minimum period, are more consistent with pure secondary nature. In order to resolve the important questions regarding possible novel sources, we undertook the higher precision measurements reported here.

In addition, one can use the charge-sign difference of antiprotons to explore effects of drift in the solar modulation. A detailed understanding of the effects of solar modulation is important to establish the existence of any primary sources. During the positive polarity phase of solar activity before 2000 the measured antiproton-to-proton $(\overline{\mathrm{p}} / \mathrm{p})$ ratio [5] showed no distinctive variation. After the reversal of the solar magnetic field in 2000, a sudden increase of the

\footnotetext{
* Corresponding author. Email address: haino@post.kek.jp (S. Haino).

1 Present address: Kamioka Observatory, ICRR, The University of Tokyo, Kamioka, Gifu 506-1205, Japan

2 Present address: Istituto Nazionale di Fisica Nucleare (INFN), Perugia 06123, Italy

3 Also at: CRESST/USRA, Columbia, MD 21044, USA

4 Present address: Physics Department, Technion - Israel Institute of Technology, Technion City, Haifa 32000, Israel.

5 Present address: Max-Planck-Institut für Physik, München 80805, Germany

6 Present address: Japan Atomic Energy Agency (JAEA), Tokai-mura, Naka-gun, Ibaraki 319-1195, Japan
} 
$\overline{\mathrm{p}} / \mathrm{p}$ ratio [6] was clearly observed. Our measurements, as well as measurements of the positron fraction $\left(\mathrm{e}^{+} /\left(\mathrm{e}^{+}+\mathrm{e}^{-}\right)\right)[15]$, generally support recent calculations $[10,16]$ incorporating steady-state drift models and charge-dependent effects of solar modulation. Protons and antiprotons have significantly different interstellar spectra and the drift directions are opposite because of the opposite charge sign. The combination of these effects implies that the $\bar{p} / p$ ratio should display a more interesting evolution [16] during 2000-2010 than it did during the 1990's. The secondary antiprotons are also useful to probe the average cosmic-ray proton spectrum over a large region of the Galaxy [17].

The BESS-Polar experiment was proposed as an advanced BESS program of long-duration balloon flights over Antarctica and has been prepared since $2001[18,19,20]$ to further investigate elementary particle phenomena in the early Universe through a precise measurement of the low-energy antiproton spectrum and to search for primary antinuclei in cosmic rays. We report here a measurement of the cosmic-ray antiproton spectrum based on 1,520 antiproton events detected during an 8.5-day flight of the BESS-Polar spectrometer performed in December 2004. We discuss the origin of cosmic-ray antiprotons, and the solar modulation effect based on the measured $\overline{\mathrm{p}} / \mathrm{p}$ ratio.

\section{Spectrometer}

The BESS-Polar superconducting spectrometer, shown in Fig. 1, has been developed to reduce material thickness along the particle trajectory and to meet the severe requirements for long duration balloon flights over Antarctica $[18,20]$. The basic instrument concepts, such as the cylindrical configuration with an open and wide acceptance and redundant measurements for particle identification, are inherited from the BESS spectrometer [21]. A very low instrumental energy cutoff for antiprotons was achieved with a new thinwalled $\left(2.46 \mathrm{~g} / \mathrm{cm}^{2} /\right.$ wall including cryostat $)$ superconducting magnet $[22,23]$ using a new high-strength aluminum-stabilized superconductor. In addition, the outer pressure vessel was eliminated and the detectors were reconfigured. Low-energy particles only have to traverse $4.5 \mathrm{~g} / \mathrm{cm}^{2}$ of instrument material to be detected, about one quarter of that in the previous BESS spectrometer $[21,24]$. With these changes, the lowest energy for antiproton detection has been reduced to $0.1 \mathrm{GeV}$ at the top of atmosphere (TOA).

A uniform field of 0.8 Tesla is produced by the superconducting coil, and its continuous operation is maintained for over 11 days with a 400-liter liquid helium reservoir. A JET-cell type drift chamber (JET) and two inner drift chambers (IDCs), which were also used in the BESS-TeV spectrometer [24,25], are placed inside the warm bore $(0.80 \mathrm{~m}$ in diameter and $1.4 \mathrm{~m}$ in length). The JET and IDCs are filled with $\mathrm{CO}_{2}$ gas, and fresh gas is circulated using a 
semi-active flow control system. Tracking of an incident particle in the $x-y$ plane (perpendicular to the magnetic field) inside the JET and IDCs is performed by fitting up to 52 hit-points, each with $150 \mu \mathrm{m}$ resolution, resulting in a magnetic-rigidity ( $R \equiv p \mathrm{c} / Z e$, momentum divided by electric charge) resolution of $0.4 \%$ at $1 \mathrm{GV}$, and a maximum detectable rigidity (MDR) of 240 GV. Tracking in the $z$ coordinate (parallel to the magnetic field direction) is done by fitting points inside IDCs measured by vernier pads with an accuracy of $1.0 \mathrm{~mm}$ and points in JET measured by charge division with an accuracy of $37 \mathrm{~mm}$. The continuous and redundant 3-dimensional tracking enables BESS-Polar to distinguish backgrounds such as tracks having interaction or scattering. A truncated mean of the integrated charges of the hit-pulses from the JET also provides an energy loss $(\mathrm{d} E / \mathrm{d} x)$ measurement with a resolution of $10 \%$.

The top and bottom scintillator hodoscopes, the time-of-flight (TOF) detectors, are used to determine the incident particle velocity, $\beta=v / \mathrm{c}$. They also make two independent $\mathrm{d} E / \mathrm{d} x$ measurements. The scintillator hodoscopes consist of 10 top and 12 bottom plastic scintillators with a cross section of $96.5 \mathrm{~mm}$ (width) $\times 10 \mathrm{~mm}$ (thickness) and with photo-multiplier tubes (PMTs) attached by acrylic light guides at each end. The timing resolution of each hodoscope is $110 \mathrm{ps}$, resulting in a $\beta^{-1}$ resolution of $3.3 \%$. In addition, a thin scintillator middle-TOF (MTOF) is installed on the lower surface of the solenoid bore to detect low energy particles which cannot penetrate the magnet wall. The MTOF consists of 64 plastic scintillator bars with a cross section of $10 \mathrm{~mm}$ (width) $\times 5 \mathrm{~mm}$ (thickness) read by multi-anode PMTs. The $\beta$ of the low energy antiprotons can be measured as a combination of top-middle TOF with a $\beta^{-1}$ resolution of $4.5 \%$ below $0.6 \mathrm{GV}(0.2 \mathrm{GeV}$ for proton and antiproton). A Cerenkov counter with silica-aerogel radiator (ACC) is installed below the magnet. The radiator is selected to have a refractive index of 1.02 in order to veto $e^{-} / \mu^{-}$backgrounds up to $4.2 \mathrm{GeV}$. The top and bottom TOF hodoscopes and the ACC operate at ambient pressure.

The event data acquisition sequence is initiated by a coincidence of signals in the top-bottom TOF or top-middle TOF hodoscopes [26]. Based on digitized detector information sent from the front-end electronics, the event data are built and recorded to onboard hard disk drives with a total capacity of 3.5 Tera Bytes. During the Antarctic flight, the data acquisition rate was around $1.4 \mathrm{kHz}$, and the dead time was $150 \mu$ s per event $(20 \%$ of the data-taking time). To supply electric power to the electronics onboard the payload, a solar cell array of $900 \mathrm{~W}$ capacity was mounted on an omni-directional octagonal frame around the payload. The total power consumption of the spectrometer was $420 \mathrm{~W}$. 


\section{Data Analysis}

\subsection{Balloon flight observation}

The first BESS-Polar long duration balloon flight over Antarctica was launched from Williams Field $\left(77^{\circ} 51.8^{\prime} \mathrm{S}, 167^{\circ} 5.4^{\prime} \mathrm{E}\right)$ near McMurdo Station on December 13, 2004 [27]. During the flight, some PMTs of the TOF hodoscopes had to be turned off because they were drawing excessive current, and the usable geometrical acceptance was consequently reduced to $73 \%$ of the design value $\left(0.157 \mathrm{~m}^{2} \mathrm{sr}\right.$ at $0.2 \mathrm{GeV}$ and $0.166 \mathrm{~m}^{2} \mathrm{sr}$ at $\left.2.0 \mathrm{GeV}\right)$. The flight was terminated on December 21 and the payload landed at the south end of the Ross Ice Shelf $\left(83^{\circ} 6.0^{\prime} \mathrm{S}, 155^{\circ} 35.4^{\prime} \mathrm{W}\right)$ after a continuous observation period of 8.5 days. The flight trajectory was close enough to the South magnetic pole that the geomagnetic cutoff rigidity was below $0.2 \mathrm{GV}$, lower than the lowest detection limit of the spectrometer. During the live data-taking time of 507,075 seconds at an average floating altitude of $38.5 \mathrm{~km}$ (residual atmosphere of $4.3 \mathrm{~g} / \mathrm{cm}^{2}$ ), $894,482,590$ cosmic-ray events were accumulated without any online event selection as 2.14 terabytes of data recorded on the hard disk drives.

\subsection{Event selection}

Since the spectrometer is cylindrically symmetric, it may be assumed that antiprotons behave exactly like protons in the instrument except for the sign of their deflection in the magnetic field and their inelastic interactions. Thus, all the selection criteria were defined based on the measured properties of protons. Initially, events were selected with (1) a single track and a downwardgoing particle fully contained in the fiducial region of the tracking volume, (2) only one or two hits each in the top and bottom TOF hodoscopes, (3) the hit position at the TOF hodoscopes consistent with the extrapolated track inside the JET and IDCs, (4) cosine of zenith angle of the incident particle larger than 0.8, and (5) either top or bottom TOF hodoscopes read by PMTs at both ends. As a consequence of (5), the effective geometrical acceptance had to be further reduced to be $40 \%$ of the design value.

The selection efficiency was estimated using a Monte Carlo (MC) simulation by applying the same selection criteria to the simulated events as to the observed data. The MC simulation was based on a GEANT3/GHEISHA code [28,29] and tuned to reproduce the results of an accelerator beam test of the previous BESS spectrometer [30] in which the detector configuration and materials are similar to the BESS-Polar spectrometer. The efficiency varied from 84.2 $\pm 5.0 \%$ at $0.2 \mathrm{GeV}$ to $90.1 \pm 5.0 \%$ at $2.0 \mathrm{GeV}$. The systematic error of the efficiency 
was determined using the accelerator beam test data. Each analog-to-digital board for TOF has an individual dead time due to the period of switching reference capacitors used for baseline subtraction. Events digitized during the dead time have incorrect charge data and were rejected to ensure the quality of the data. The efficiency of surviving this cut was estimated as $77.8 \pm 0.1 \%$ at $0.2 \mathrm{GeV}$ and $78.8 \pm 0.1 \%$ at $2.0 \mathrm{GeV}$. Here and in the selections described below, a sample of the proton data has been used to determine the efficiencies.

The following track quality cuts were applied: (1) the reduced $\chi^{2}$ in the $x-y$ and $y-z$ track fitting to be less than $5,(2)$ the fitting error on the curvature (inverse rigidity) to be less than $0.015 \mathrm{GV}^{-1},(3)$ the track fitting path length to be longer than $500 \mathrm{~mm}$, and (4) the residual between hit position at TOF obtained from the time difference in two PMTs and the extrapolated track of the JET to be less than $50 \mathrm{~mm}$. The quality cut efficiency was estimated as $94.7 \pm 0.2 \%$ at $0.2 \mathrm{GeV}$ and $91.7 \pm 0.1 \%$ at $2.0 \mathrm{GeV}$.

\subsection{Particle identification}

Proton and antiproton candidates were identified with a combination of a charge selection and a mass selection as follows: (1) Particle charge, $Z$ is identified by $\mathrm{d} E / \mathrm{d} x$ measurements. The $\mathrm{d} E / \mathrm{d} x$ measurements with both top and bottom TOF hodoscopes were required to be inside a band defined as a function of rigidity corresponding to singly-charged particles. (2) Particle mass $m$

is reconstructed with rigidity $R$, velocity $\beta$ and $Z$ as $m=Z e R \sqrt{1 / \beta^{2}-1}$. It was required that $1 / \beta$ be inside a band defined as a function of rigidity as shown in Fig. 2, so that the reconstructed mass was consistent with that of a proton or antiproton. The $\mathrm{d} E / \mathrm{d} x$ and $\beta$ bands for antiprotons were defined in the same way as for protons except for the rigidity sign. The selection efficiency of each $\mathrm{d} E / \mathrm{d} x$ band cut was estimated as a fraction of the number of selected events among a proton sample selected by the other independent $\mathrm{d} E / \mathrm{d} x$ selections. The net efficiency for the two $\mathrm{d} E / \mathrm{d} x$ band cuts was $96.5 \pm 0.1 \%$ at $0.2 \mathrm{GeV}$ and $96.4 \pm 0.1 \%$ at $2.0 \mathrm{GeV}$. The selection efficiency of the $1 / \beta$ band cut was estimated as $99.5 \pm 0.2 \%$ at $0.2 \mathrm{GeV}$ and $97.0 \pm 0.1 \%$ at $2.0 \mathrm{GeV}$.

In order to eliminate $\mathrm{e}^{-}$and $\mu^{-}$backgrounds that mimic relativistic antiproton candidates with $\beta>0.9$, Cerenkov veto cuts were applied to select (3) the particle trajectory to be inside an aerogel fiducial volume, and (4) the Čerenkov outputs to be less than a threshold. The rejection factor was estimated as $(8.9 \pm 0.2) \times 10^{2}$ using a relativistic proton sample with rigidity larger than $20 \mathrm{GV}$. The efficiency including a loss of fiducial volume by $18 \%$ was estimated as $75.8 \pm 0.1 \%$ at $0.4 \mathrm{GeV}$ and $62.3 \pm 0.1 \%$ at $2.0 \mathrm{GeV}$.

Because of the relativistic rise of $\mathrm{d} E / \mathrm{d} x$ in the JET gas, ultra-relativistic 
particles $(\beta \rightarrow 1)$ have $\mathrm{d} E / \mathrm{d} x$ about 1.4 times higher than minimum ionizing particles. A tight cut on $\mathrm{d} E / \mathrm{d} x$ measured with the JET can eliminate part of the $\mathrm{e}^{-}$and $\mu^{-}$backgrounds. (5) The $\mathrm{d} E / \mathrm{d} x$ measurements with the JET were required to be inside a band defined as a function of rigidity. The rejection factor was estimated to be $1.75 \pm 0.03$ at $2.0 \mathrm{GeV}$ and $4.85 \pm 0.08$ at $4.0 \mathrm{GeV}$, as a fraction of the rejected events among the $\mathrm{e}^{-}$and $\mu^{-}$sample which was obtained with the same selection criteria as antiprotons excluding Cerenkov veto cuts. The efficiency was estimated to be $98.2 \pm 0.1 \%$ at $2.0 \mathrm{GeV}$ and $96.9 \pm 0.1 \%$ at $4.0 \mathrm{GeV}$.

Following the particle identification procedure, 1,512 antiproton candidates were identified above $0.2 \mathrm{GeV}$ as shown in Fig. 2. Antiproton candidates that have wrongly reconstructed $\beta$ and lie above the selection band were rejected by the $1 / \beta$ band cuts. The antiproton flux was obtained by correcting for the cut efficiency estimated using the proton sample. The event sample obtained with the antiproton selection criteria excluding Cerenkov veto cuts and the JET $\mathrm{d} E / \mathrm{d} x$ cut consists of mostly $(>99.8 \%) \mathrm{e}^{-}$and $\mu^{-}$background events. The number of such events divided by the total rejection factors defined above was used as an estimation of the number of contaminating backgrounds within the antiproton selection band. The estimated number of background events (and the ratio to antiproton candidates) was $18.7 \pm 0.3(8.5 \pm 0.1 \%)$ in the $1.7-$ $2.1 \mathrm{GeV}$ band and $8.1 \pm 0.1(4.0 \pm 0.1 \%)$ in the $3.4-4.2 \mathrm{GeV}$ band. In the region $-1.5 \mathrm{GV}<R<0$ and $0.9<1 / \beta<1.1$, the remaining background events can be selected with only the $1 / \beta$ band cut and the number of such events was 235 , which was consistent with the estimated number, $243 \pm 11$.

Albedo and spillover from positive rigidity particles were negligible because of the high $\beta^{-1}$ and rigidity resolutions. To check against the "re-entrant albedo" background, we confirmed that the trajectories of all antiprotons could be traced numerically through the Earth's geomagnetic field back to the outside of the geomagnetic field [31,32].

\subsection{Selection with top-middle TOF below $0.2 \mathrm{GeV}$}

Some of the antiprotons below $0.2 \mathrm{GeV}$ cannot reach the bottom TOF hodoscope due to energy and annihilation losses. To analyze such low energy events, we defined another set of selection criteria with a combination of the top and middle TOF hodoscopes. They are basically the same as those used with the top and bottom TOF, but the Čerenkov veto cuts were not applied since at low energies antiproton candidates are well separated from $\mathrm{e}^{-}$and $\mu^{-}$backgrounds using only the $1 / \beta$ band cut. Using the top-middle TOF selection criteria, 8 antiproton candidates were identified in the energy range $0.1-0.2 \mathrm{GeV}$. 
Stopping antiprotons can generate secondaries in the upward direction that make additional tracks inside the JET. Such events are rejected by the singletrack selection. To recover such events, a search was made for antiproton candidates among multi-track events. The major backgrounds for this search are upward moving proton secondaries generated in the lower half of the spectrometer from interactions of high energy protons. Upward moving protons can usually be distinguished with the measured $\beta$, but if the incident proton and the upward moving secondary hit the same top TOF counter, the $\beta$ is not correctly measured. Such events were rejected by applying the following cuts: (1) Stopping antiprotons cannot generate high energy secondaries because of the kinematical restriction. Hence for this search rigidities of all the tracks inside the JET were required to be less than $1 \mathrm{GV}$. (2) It was required that the distance between the two tracks at the top TOF to be larger than $150 \mathrm{~mm}$. The efficiency of these cuts was estimated as $72.1 \pm 2.1 \%$ by applying the same cuts to MC generated antiproton events. A total of 31 multi-track antiproton candidates were found, but all of them were rejected by the cuts. According to the MC study, the total antiproton detection efficiency was increased by $11.1 \%$ at $0.1 \mathrm{GeV}$ by accepting multi-track events. This increase was treated as a systematic error, but it was smaller than the statistical error $(+41 \% /-34 \%)$ of single-track antiproton candidates below $0.2 \mathrm{GeV}$.

\subsection{Flux determination}

After the antiproton candidates were identified, energy-dependent corrections were applied for backgrounds and detection efficiency. Then the absolute flux at the top of the instrument (TOI) was obtained by taking account of energy loss inside the spectrometer, live time, and geometrical acceptance. The energy of each particle at TOI was calculated by summing up the ionization energy losses inside the instrument as determined by tracing back the event trajectory. The effective geometrical acceptance was estimated using the MC code GEANT3 [28] and the simulation technique [33] to be $0.114 \pm 0.001 \mathrm{~m}^{2} \mathrm{sr}$ at $0.2 \mathrm{GeV}$ and $0.121 \pm 0.001 \mathrm{~m}^{2} \mathrm{sr}$ at $2.0 \mathrm{GeV}$. The error arising from uncertainty in the detector alignment was estimated to be $1 \%$.

In order to obtain the flux at the top of the atmosphere (TOA), we applied a correction for survival probability of the flux reaching TOI from TOA, and a subtraction of the secondary component produced within the overlying atmosphere. The survival probability was estimated as $89.1 \pm 2.0 \%$ at $0.2 \mathrm{GeV}$ and $92.4 \pm 2.0 \%$ at $2.0 \mathrm{GeV}$ based on total interaction lengths of $32.7 \pm 0.7 \mathrm{~g} / \mathrm{cm}^{2}$ at $0.2 \mathrm{GeV}$ and $59.9 \pm 1.2 \mathrm{~g} / \mathrm{cm}^{2}$ at $2.0 \mathrm{GeV}[34,35]$.

The atmospheric secondary antiproton flux was estimated by solving simultaneous transport equations [34,36]. At low energies below $1 \mathrm{GeV}$, there exists a 
significant contribution of non-annihilating inelastic interactions or so-called "tertiary" antiprotons. The interaction length used was based on Stephen's model [35], and the energy distribution of the tertiary production was tuned to reproduce the atmospheric antiproton flux measurements $[37,38]$. The amount of secondary subtraction was $11.8 \pm 1.7 \%$ at $0.2 \mathrm{GeV}$ and $28.6 \pm 4.1 \%$ at $2.0 \mathrm{GeV}$. The relative error of $14.3 \%$ is composed of uncertainty in the residual air depth $(5.0 \%)$, in the cross section of primary cosmic rays with air nuclei $(8.9 \%)$, and in the tertiary production $(10.0 \%)$.

\section{Results and Discussions}

We obtained the antiproton flux at the TOA in the kinetic energy range 0.10-4.20 GeV as shown in Fig. 3 and tabulated in Table 1. The lowest energy was determined by the detector cutoff energy, and the highest energy was determined by the antiproton threshold energy of the aerogel Cerenkov counter. The overall uncertainties including statistical and systematic errors are $-34.1 \% /+41.8 \%$ at $0.16 \mathrm{GeV}$ and $\pm 10.9 \%$ at $3.7 \mathrm{GeV}$ with the given energy bin width. The statistical errors are dominant over the systematic errors below $1.4 \mathrm{GeV}$. Owing to the long duration observation the statistical errors were improved from the previous measurements with BESS, carried out on conventional 1 or 2 day balloon flights.

Fig. 3 also shows the results from previous BESS flights around solar minimum $(95+97)[3,4]$ and maximum (2000) [6], which are compared with four theoretical calculations. The solid curves are calculations of secondary antiproton spectra with the Standard Leaky Box (SLB) model modulated with a steady state drift model [16], in which the modulation is characterized by a tilt angle of the heliospheric current sheet and the Sun's magnetic polarity (denoted as $+/-$ ). The dashed curves are calculations with the Diffusion plus Convection (DC) model modulated with a drift model [10,42]. Three tilt angles, $10^{\circ}(+), 70^{\circ}(-)$, and $30^{\circ}(-)$ roughly correspond to the measurements with BESS(95+97), BESS(2000) and BESS-Polar(2004), respectively [43,44]. The dotted curves are calculations with the DC model [10] modulated with a standard spherically symmetric approach [39], in which the modulation is characterized by a single parameter $(\phi)$ irrespective of the Sun's polarity. For each measurement, $\phi$ was obtained by fitting that proton spectrum measured by BESS, assuming the interstellar spectrum in Ref. [40]. Three values of $\phi, 550 \mathrm{MV}, 1400 \mathrm{MV}$, and $850 \mathrm{MV}$ correspond to the measurements with BESS(95+97), BESS(2000) and BESS-Polar(2004), respectively. The dash-dot curves are calculations of antiproton spectra from evaporation of Primordial Black Holes $(\mathrm{PBH})$ with an explosion rate of $0.4 \times 10^{-2} \mathrm{pc}^{-3} \mathrm{yr}^{-1}[12,41]$ modulated by the same spherically symmetric approach with $\phi$ of $550 \mathrm{MV}$ and $850 \mathrm{MV}$. The expected signal from the PBH is modulated more than the sec- 
ondary antiproton spectrum because of its spectral shape having the peak in the low energy region. While the BESS $(95+97)$ data were suggestive of an excess flux at energies below $400 \mathrm{MeV}$, we do not find further evidence in the new BESS-Polar data presented here, even with the extended energy range.

The $\overline{\mathrm{p}} / \mathrm{p}$ ratio can provide a useful probe to study solar modulation and its charge-sign dependence. The BESS collaboration has tracked this ratio through most of a solar cycle. Fig. 4 shows the $\bar{p} / p$ ratio obtained with the BESS-Polar measurement together with the results from previous BESS flights around solar minimum $(95+97)[3,4]$ and maximum (2000) [6], which are compared with the same three calculations of secondary antiproton spectra as shown in Fig. 3. Fig. 5 shows time variations of $\bar{p} / p$ ratio at three different energies, $0.3 \mathrm{GeV}, 1.0 \mathrm{GeV}$, and $1.9 \mathrm{GeV}$, each compared with the same calculations as shown in Fig. 4.

Since the $\overline{\mathrm{p}} / \mathrm{p}$ ratios obtained using drift models are given as a function of tilt angle, they were converted into those as a function of time by taking the tilt angle as the mean position of the monthly variation of the maximum latitudinal extent of the current sheet $[43,44]$. The time variation of $\bar{p} / p$ ratios by the spherically symmetric approach were estimated by using a linear relation between $\phi_{B E S S}$ and $N_{C L}$, where $\phi_{B E S S}$ was obtained by fitting each proton spectrum measured with 6 BESS flights [40] and $N_{C L}$ is the monthly averaged count rates of the Climax neutron monitor $[45] 7$.

Figs. 4 and 5 show that drift models and the symmetric model reproduce equally well the stable $\overline{\mathrm{p}} / \mathrm{p}$ ratio during the positive phase. The sudden increase of the ratio observed by BESS measurements after the positive-to-negative solar field reversal is better reproduced by the drift models at energies below $1 \mathrm{GeV}$. On the other hand, during the negative phase, where the $\overline{\mathrm{p}} / \mathrm{p}$ ratio depends on the tilt angles more than during the positive phase, the two drift model calculations $[10,16]$ significantly differ from each other. We found that the difference mainly comes from the difference in the modulation of protons, which in the negative phase should come from a "horizontal" direction in heliosphere, i.e. along the current sheet. This would imply that the model of particles behavior along the current sheet is not yet established well. Our data prefer calculations by Bieber et al. [16] but those with the spherical model are also preferred. In addition, Fig. 3 shows that the spherical model reproduces antiproton spectra of all the three BESS measurements better than drift models. This implies that the charge-sign dependence in the modulation is not so significant during the negative phase. The spherical model is still the best one to reproduce measurements.

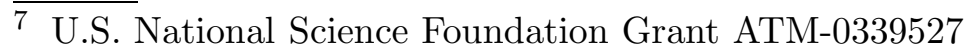




\section{Conclusion}

The first BESS-Polar experiment was carried out in Antarctica in December 2004, a transient period before the solar minimum in 2007. Using a new spectrometer with reduced material thickness and a long duration balloon flight near the Earth's south magnetic pole, the lowest energy limit of the antiproton flux was extended down to $0.1 \mathrm{GeV}$, and the statistics were improved compared with the previous BESS experiments. The series of BESS measurements have enabled a crucial test of models of the solar modulation. Drift models can reproduce the drastic behavior of the $\overline{\mathrm{p}} / \mathrm{p}$ ratio around the solar field reversal, but for the negative phase the spherical model is still best able to reproduce most of the measurements. BESS data should motivate further development of the drift models with more realistic parameters and time dependence. For a more extensive future search for cosmic-ray antiprotons of primordial origin the BESS-Polar result provides an important baseline measurement of the secondary antiproton spectrum, which will be compared with a spectrum measured in December 2007 through January 2008, by the second Antarctic flight, during the solar minimum period.

\section{Acknowledgements}

The authors thank NASA Headquarters for continuous encouragement in this U.S.-Japan cooperative project. Sincere thanks are expressed to the NASA Balloon Programs Office at GSFC/WFF and to the NASA Columbia Scientific Balloon Facility for their experienced support. They also thank ISAS/JAXA and KEK for their continuous support and encouragement. Special thanks go to the National Science Foundation (NSF), USA, and Raytheon Polar Service Company for their professional support in the USA and in Antarctica. The authors would thank the BESS-Polar II collaborators M. Hasegawa, A. Horikoshi, K. Sakai, and N. Thakur for their contribution to the instrument performance evaluation and further cooperation. The BESS-Polar experiment is being carried out as a Japan-U.S. collaboration. It is supported by MEXT grants (KAKENHI-13001004, 15340077, and 181040006) in Japan, and by NASA grants in the USA.

\section{References}

[1] K. Yoshimura, et al., Phys. Rev. Lett. 75 (1995) 3792.

[2] A. Moiseev, et al., Astrophys. J. 474 (1997) 479. 
[3] H. Matsunaga, et al., Phys. Rev. Lett. 81 (1998) 4052.

[4] S. Orito, et al., Phys. Rev. Lett. 84 (2000) 1078.

[5] T. Maeno, et al., Astropart. Phys. 16 (2001) 121.

[6] Y. Asaoka, et al., Phys. Rev. Lett. 88 (2002) 051101.

[7] S. Haino, et al., Proc. 29th Int. Cosmic Ray Conf. (Pune) 3 (2005) 13.

[8] T. K. Gaisser and R. K. Schaffer, Astrophys. J. 394 (1992) 174.

[9] T. Mitsui, Ph.D. thesis, University of Tokyo, 1996.

[10] I .V. Moskalenko, et al., Astrophys. J. 565 (2002) 280.

[11] S. W. Hawking, Commun. Math. Phys. 43 (1975) 199.

[12] K. Maki, T. Mitsui and S. Orito, Phys. Rev. Lett. 76 (1996) 3474.

[13] T. Mitsui, K. Maki and S. Orito, Phys. Lett. B 389 (1996) 169.

[14] L. Bergström, et al., Astrophys. J. 526 (1999) 215.

[15] J. Clem and P. Evenson, J. Geophys. Res. 109 (2004) A07107.

[16] J. W. Bieber, et al., Phys. Rev. Lett. 83 (1999) 674.

[17] A. W. Strong, I .V. Moskalenko and O. Reimer, Astrophys. J. 613 (2004) 962.

[18] A. Yamamoto, et al., Adv. Space Res. 30(5) (2002) 1253.

[19] J. W. Mitchell, et al., Nucl. Phys. B (Proc. Suppl.) 134 (2004) 31.

[20] T. Yoshida, et al., Adv. Space Res. 33(10) (2004) 1755.

[21] Y. Ajima, et al., Nucl. Instrum. Methods A 443 (2000) 71.

[22] A. Yamamoto, et al., IEEE Trans. Appl. Superconduct. 12(1) (2002) 438.

[23] Y. Makida, et al., IEEE Trans. Appl. Superconduct. 16(2) (2006) 477.

[24] S. Haino, et al., Nucl. Instrum. Methods A 518 (2004) 167.

[25] S. Haino, et al., Phys. Lett. B 594 (2004) 35.

[26] M. Sasaki, et al., Proc. 29th Int. Cosmic Ray Conf. (Pune) 3 (2005) 421.

[27] T. Yoshida, et al., Proc. 29th Int. Cosmic Ray Conf. (Pune) 3 (2005) 33.

[28] R. Brun, et al., GEANT3.21-Detector Description and Simulation Tool, CERN Program Library, Long Write up W5013.

[29] H. Fesefeldt, PITHA 85/02, Aachen, (1985).

[30] Y. Asaoka, et al., Nucl. Instrum. Methods A 489 (2001) 170.

[31] M. Honda, et al., Phys. Rev. D 70 (2004) 043008. 
[32] International Geomagnetic Reference Field, 9th generation (2003) $<$ http://www.ngdc.noaa.gov/IAGA/vmod/igrf.html $>$.

[33] J. D. Sullivan, Nucl. Instrum. Methods 95 (1971) 5.

[34] S. A. Stephens, Astropart. Phys. 6 (1997) 229.

[35] S. A. Stephens, Proc. 29th Int. Cosmic Ray Conf. (Pune) 3 (2005) 169.

[36] P. Papini, et al., Nuovo Cimento 19C (1996) 367.

[37] K. Yamato, et al., Phys. Lett. B 632 (2003) 475.

[38] T. Sanuki, et al., Phys. Lett. B 577 (2003) 10.

[39] L. A. Fisk, J. Geophys. Res. 76 (1971) 221.

[40] Y. Shikaze, et al., Astropart. Phys. 28 (2007) 154.

[41] K. Yoshimura, et al., Adv. Space Res. 27(4) (2001) 693.

[42] I .V. Moskalenko, Private communication, 2007.

[43] X. Zhao and J. T. Hoeksema, Adv. Space Res. 16(9) (1995) 181.

[44] J. T. Hoeksema, Space Science Reviews 72 (1995) 137; The Wilcox Solar Observatory, <http://wso.stanford.edu/Tilts.html $>$.

[45] University of New Hampshire Neutron Monitor Datasets, $<$ http://ulysses.sr.unh.edu/NeutronMonitor/neutron_mon.html $>$.

[46] K. Yoshimura, Adv. Space Res. in press (2007). 
Table 1

Antiproton flux and $\overline{\mathrm{p}} / \mathrm{p}$ ratio at the top of atmosphere with statistical (first) and systematic (second) errors. $N_{\overline{\mathrm{p}}}$ and $N_{B G}$ are the number of observed antiprotons and estimated background events, respectively.

\begin{tabular}{cccccc}
\hline \hline \multicolumn{2}{c}{$\begin{array}{c}\text { Kinetic energy }(\mathrm{GeV}) \\
\text { range }\end{array}$} & mean & $N_{\overline{\mathrm{p}}}$ & $N_{B G}$ & $\begin{array}{c}\overline{\mathrm{p}} \text { flux } \\
\left(\mathrm{m}^{-2} \mathrm{sr}^{-1} \mathrm{~s}^{-1} \mathrm{GeV}^{-1}\right)\end{array}$ \\
\hline $0.10-0.18$ & 0.16 & 8 & 0.0 & $4.37_{-1.47-0.25}^{+1.81+0.25} \times 10^{-3}$ & $\overline{\mathrm{p}} / \mathrm{p}$ ratio \\
$0.18-0.28$ & 0.22 & 15 & 0.0 & $4.53_{-1.11-0.27}^{+1.31+0.27} \times 10^{-3}$ & $8.28_{-2.39-0.64}^{+2.03+0.64} \times 10^{-6}$ \\
$0.28-0.40$ & 0.34 & 24 & 0.0 & $6.67_{-1.30-0.27}^{+1.48+0.27} \times 10^{-3}$ & $9.49_{-2.10-0.40}^{+1.85+0.40} \times 10^{-6}$ \\
$0.40-0.56$ & 0.48 & 23 & 0.1 & $3.94_{-0.80-0.30}^{+0.91+0.30} \times 10^{-3}$ & $5.18_{-1.20-0.38}^{+1.06+0.38} \times 10^{-6}$ \\
$0.56-0.70$ & 0.65 & 37 & 0.2 & $8.27_{-1.39-0.47}^{+1.53+0.47} \times 10^{-3}$ & $1.10_{-0.20-0.06}^{+0.18+0.06} \times 10^{-5}$ \\
$0.70-0.88$ & 0.79 & 55 & 0.6 & $9.82_{-1.29-0.59}^{+1.41+0.59} \times 10^{-3}$ & $1.38_{-0.20-0.08}^{+0.18+0.08} \times 10^{-5}$ \\
$0.88-1.10$ & 1.00 & 84 & 3.3 & $1.17_{-0.13-0.07}^{+0.14+0.07} \times 10^{-2}$ & $1.83_{-0.22-0.11}^{+0.21+0.11} \times 10^{-5}$ \\
$1.10-1.37$ & 1.23 & 143 & 10.8 & $1.64_{-0.14-0.12}^{+0.15+0.12} \times 10^{-2}$ & $2.94_{-0.27-0.20}^{+0.26+0.20} \times 10^{-5}$ \\
$1.37-1.72$ & 1.54 & 198 & 18.2 & $1.84_{-0.14-0.13}^{+0.15+0.13} \times 10^{-2}$ & $4.00_{-0.33-0.28}^{+0.31+0.28} \times 10^{-5}$ \\
$1.72-2.15$ & 1.92 & 220 & 18.7 & $1.62_{-0.11-0.13}^{+0.11+0.13} \times 10^{-2}$ & $4.42_{-0.30-0.33}^{+0.30+0.33} \times 10^{-5}$ \\
$2.15-2.68$ & 2.40 & 233 & 15.1 & $1.40_{-0.09-0.12}^{+0.09+0.12} \times 10^{-2}$ & $5.01_{-0.33-0.41}^{+0.33+0.41} \times 10^{-5}$ \\
$2.68-3.36$ & 3.01 & 276 & 11.9 & $1.47_{-0.09-0.11}^{+0.09+0.11} \times 10^{-2}$ & $7.17_{-0.43-0.54}^{+0.43+0.54} \times 10^{-5}$ \\
$3.36-4.20$ & 3.68 & 204 & 8.1 & $1.10_{-0.08-0.09}^{+0.08+0.09} \times 10^{-2}$ & $7.46_{-0.52-0.60}^{+0.52+0.60} \times 10^{-5}$ \\
\hline \hline
\end{tabular}




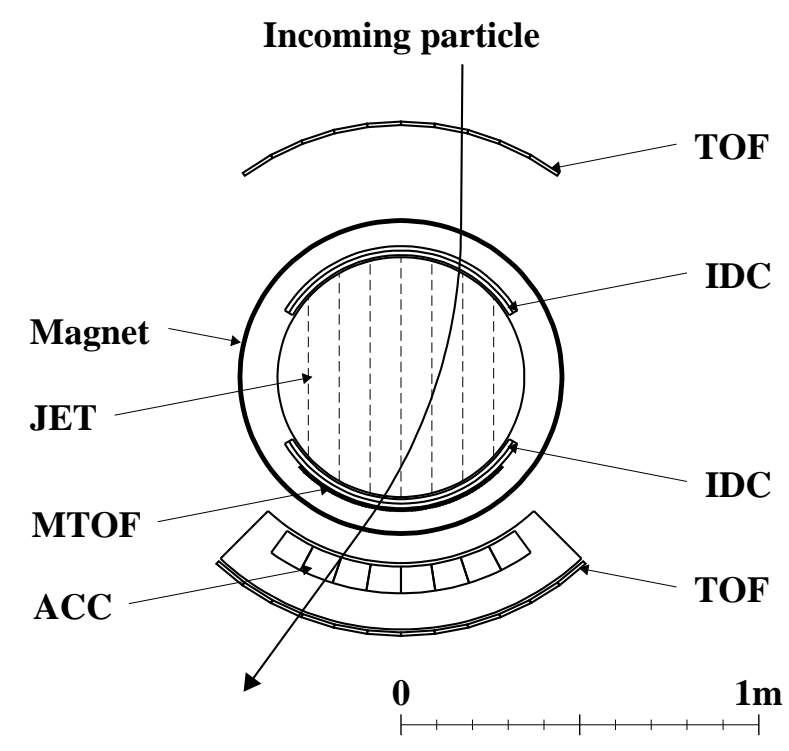

Fig. 1. Cross-sectional view of the BESS-Polar spectrometer. 


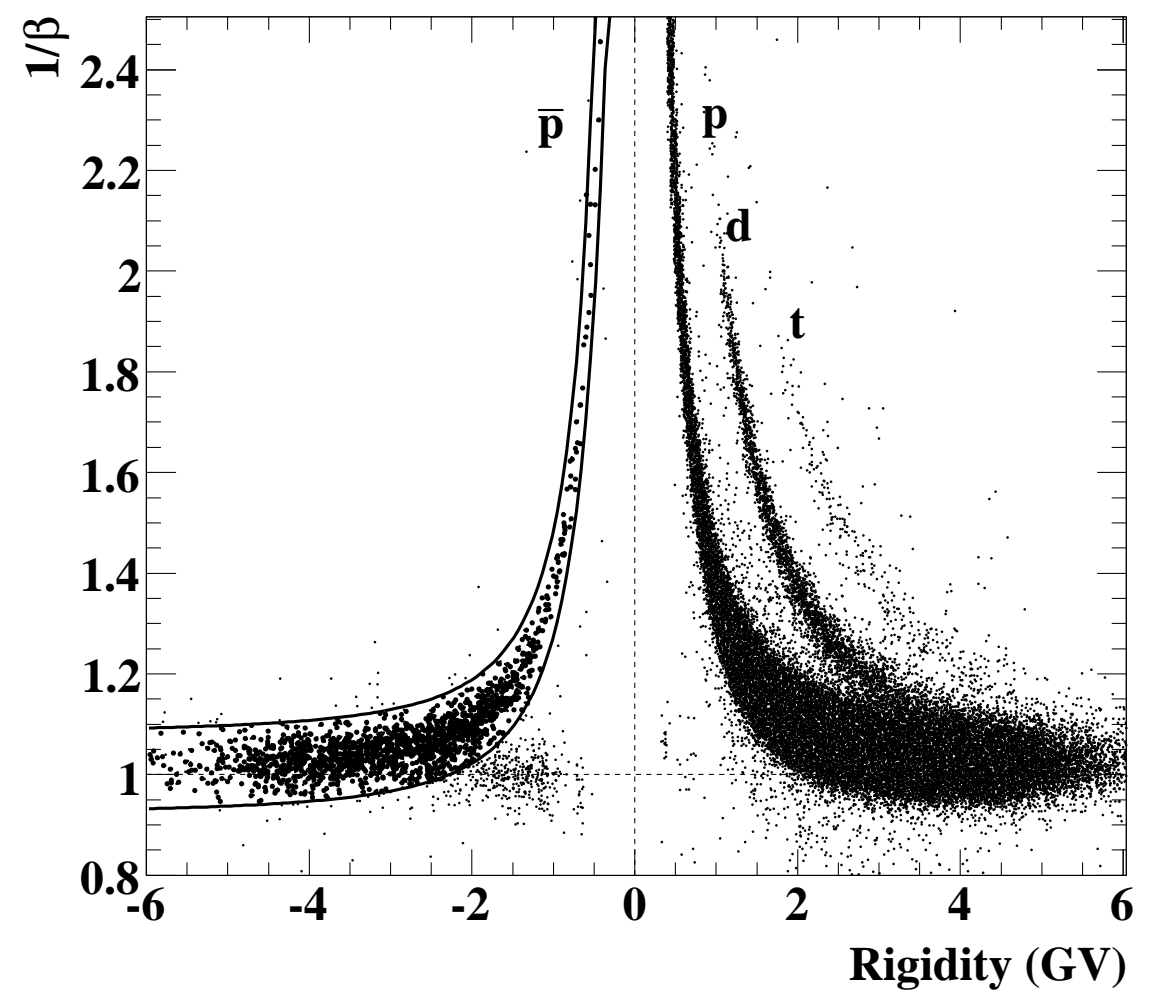

Fig. 2. The $\beta^{-1}$ versus rigidity plot, and antiproton selection band. The same band but opposite rigidity sign is applied to select protons. For the negative rigidity, all the events after Cerenkov veto cuts and JET $\mathrm{d} E / \mathrm{d} x$ cut are shown. For the positive rigidity, $0.5 \%$ of the events after Čerenkov veto cuts are shown. Antiproton candidates are shown with dots of larger size. 


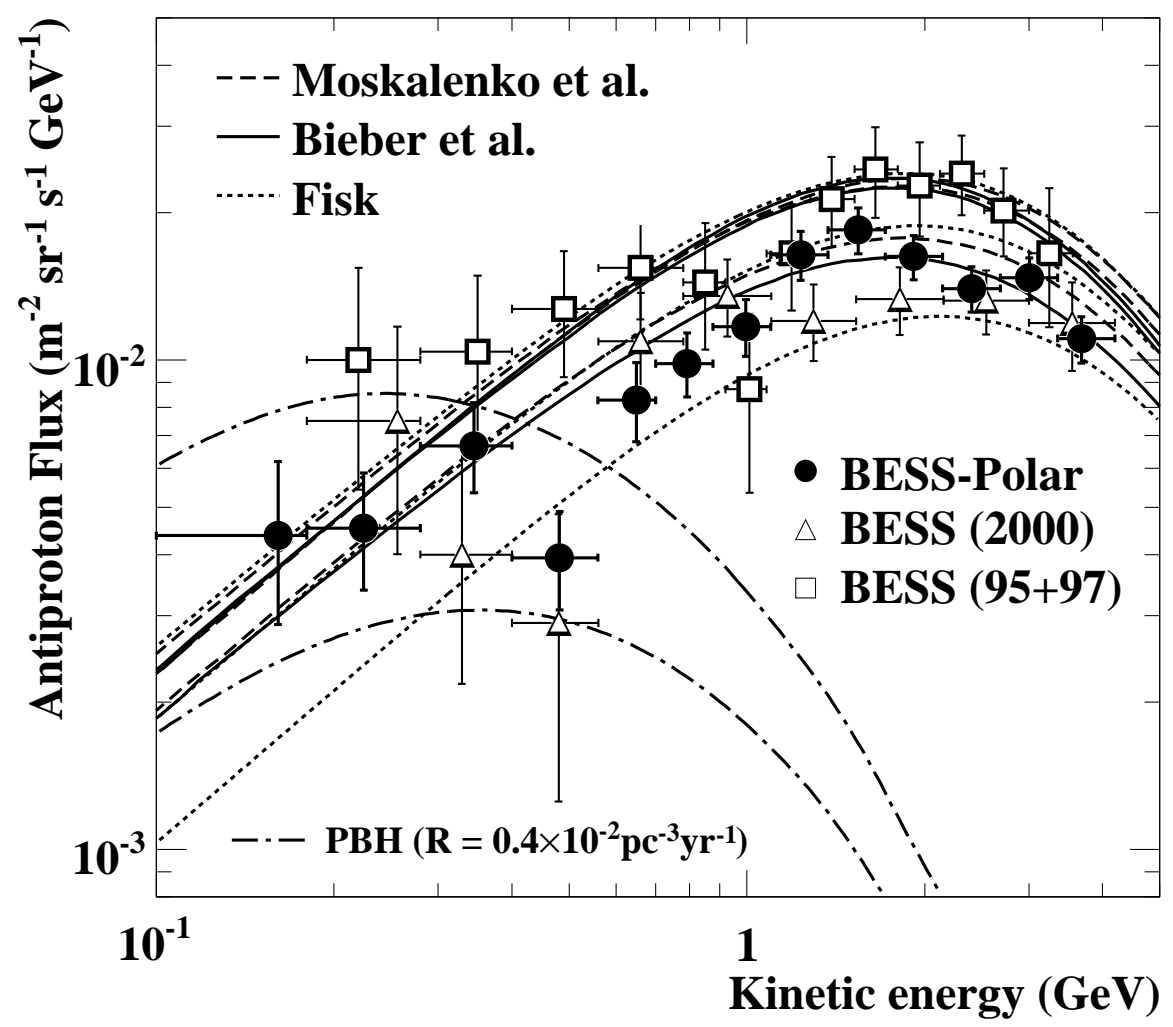

Fig. 3. Antiproton flux at the top of the atmosphere obtained with the first BESS-Polar flight together with results from previous BESS flights around solar minimum $(95+97)[3,4]$ and maximum (2000) [6]. The solid curves are calculations of secondary antiproton spectra with the Standard Leaky Box (SLB) model modulated with a steady state drift model [16] by solar tilt angles and magnetic polarities of (from top to bottom, the first two are very close) $10^{\circ}(+), 10^{\circ}(-)$, and $70^{\circ}(-)$. The dashed curves are calculations with the Diffusion plus Convection (DC) model [10] modulated by (from top to bottom, the first two are very close) $10^{\circ}(+), 30^{\circ}(-)$, and $70^{\circ}(-)$. The dotted curves are calculations with the DC model [10] modulated with a spherically symmetric model [39] by (from top to bottom) $550 \mathrm{MV}, 850 \mathrm{MV}$, and $1400 \mathrm{MV}$. The dash-dot curves are calculations of antiproton spectra from evaporation of primordial black holes with an explosion rate of $0.4 \times 10^{-2} \mathrm{pc}^{-3} \mathrm{yr}^{-1}$ modulated by $550 \mathrm{MV}$ (top) and $850 \mathrm{MV}$ (bottom) [12,41]. 


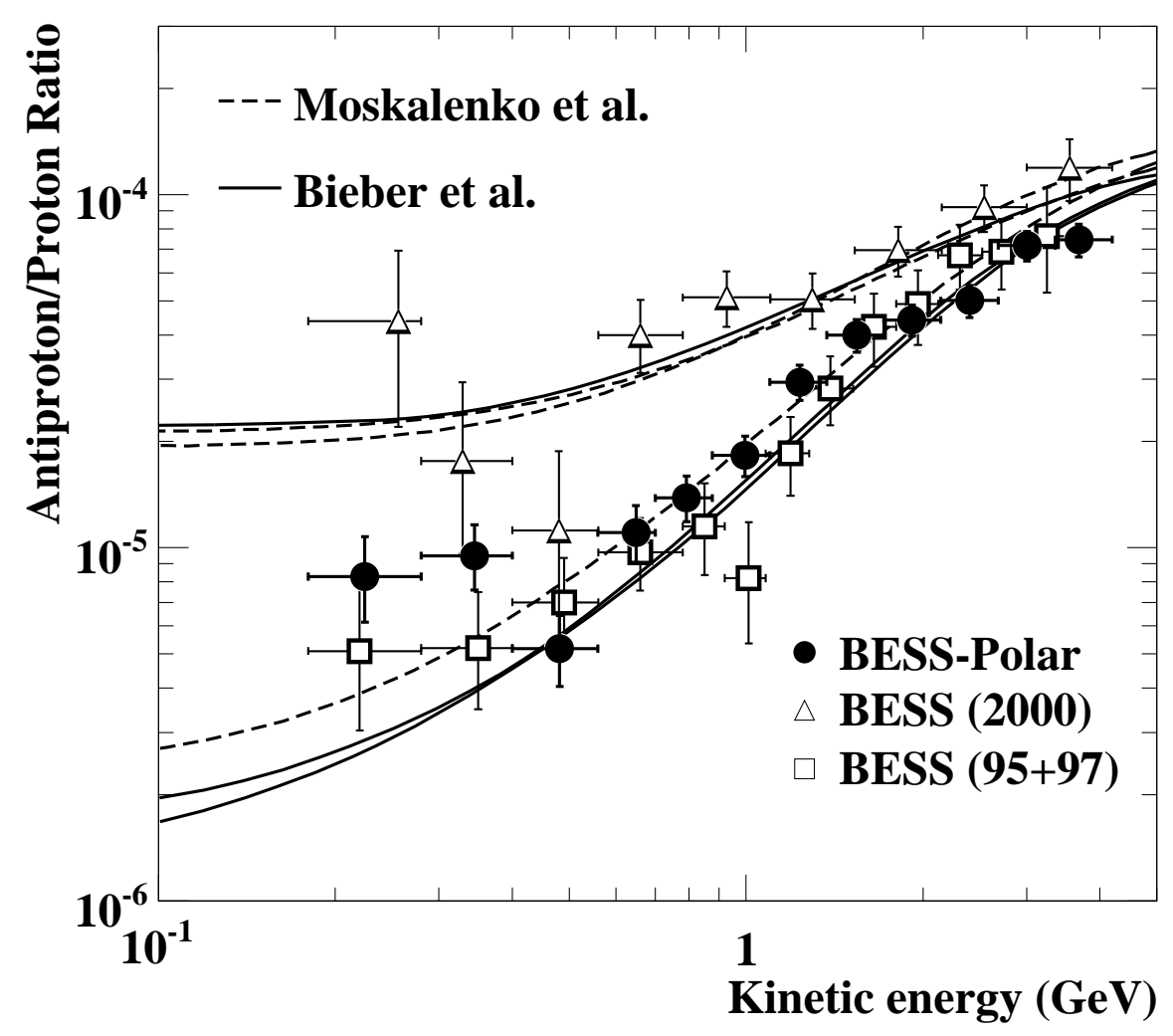

Fig. 4. $\overline{\mathrm{p}} / \mathrm{p}$ ratio obtained with the first BESS-Polar flight together with results from previous BESS flights around solar minimum $(95+97)$ [3,4] and maximum (2000) [6]. The solid curves are calculations with the Standard Leaky Box (SLB) model modulated with a steady state drift model [16] by solar tilt angles and magnetic polarities of (from bottom to top, the first two are very close) $10^{\circ}(+), 10^{\circ}(-)$, and $70^{\circ}(-)$. The dashed curves are calculations with the Diffusion plus Convection (DC) model [10] modulated by (from bottom to top, the last two are very close) $10^{\circ}(+), 30^{\circ}(-)$, and $70^{\circ}(-)$. 


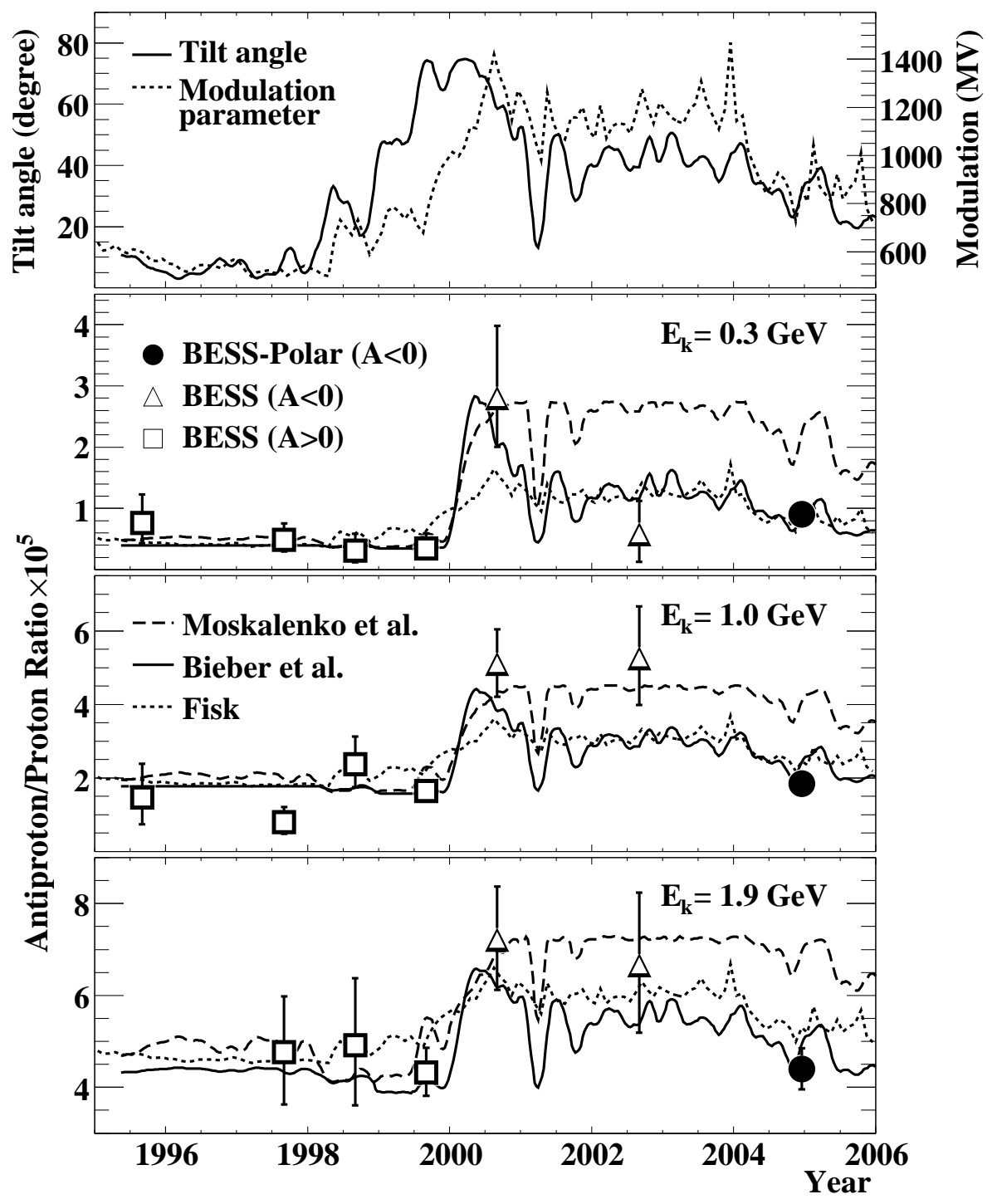

Fig. 5. The top panel shows time variations of the tilt angle $[43,44]$ shown by a solid curve and modulation parameter for the spherically symmetric model [39] shown by a dotted curve. The modulation parameter values continuous over time were estimated by using its linear relation with Climax neutron monitor data [45]. The linear relation was established using the modulation parameter for each BESS flight obtained by fitting the BESS proton spectrum. The other three panels show time variations of the $\overline{\mathrm{p}} / \mathrm{p}$ ratio at $0.3 \mathrm{GeV}$ (2nd), $1.0 \mathrm{GeV}(3 \mathrm{rd})$, and $1.9 \mathrm{GeV}$ (bottom). The data of $\overline{\mathrm{p}} / \mathrm{p}$ ratio are compared with time variations predicted by two drift models shown by solid curves from Bieber et al. [16] and dashed curves from Moskalenko et al. [10], and with the spherically symmetric moduation by Fisk [39] shown by dotted curves. 